Note: This is a draft of a paper submitted for publication. Contents of this paper should not be quoted or referred to without permission of the author(s).

To Appear in the Proceedings of The $18^{\text {th }}$ International Conference on Thermoelectrics (Invited Paper given in a Plenary Session)

\title{
Finding New Thermoelectric Compounds Using Crystallographic Data: Atomic Displacement Parameters
}

\author{
B. C. Sales, B. C. Chakoumakos, D. Mandrus, and J. W. Sharp ${ }^{1}$ \\ Solid State Division, Oak Ridge National Laboratory \\ P.O. Box 2008, Oak Ridge, Tennessee 37831-6056 \\ ${ }^{1}$ Marlow Industries, Dallas, Texas 75238
}

\begin{abstract}
"The submitted manuscript has been authored by a contractor of the U.S. Govemment under contract No. DE-AC05-960R22464. Accordingly, the U.S. Government retains a nonexclusive, royalty-free license to publish or reproduce the published form of this contribution, or allow others to do so, for U.S. Govemment purposes.
\end{abstract}

\author{
prepared by \\ SOLID STATE DIVISION \\ OAK RIDGE NATIONAL LABORATORY \\ Managed by \\ LOCKHEED MARTIN ENERGY RESEARCH CORP. \\ under \\ Contract No. DE-AC05-96OR22464 \\ with the \\ U.S. DEPARTMENT OF ENERGY \\ Oak Ridge, Tennessee
}

September, 1999 


\title{
Finding New Thermoelectric Compounds Using Crystallographic Data: Atomic Displacement Parameters
}

\author{
B. C. Sales ${ }^{1}$, B. C. Chakoumakos ${ }^{1}$, D. Mandrus ${ }^{1}$, and J. W. Sharp ${ }^{2}$ \\ ${ }^{1}$ Solid State Division, Oak Ridge National Laboratory, Oak Ridge, TN 37831 \\ ${ }^{2}$ Marlow Industries, Dallas, TX 75238
}

\begin{abstract}
A new structure-property relationship is discussed which links atomic displacement parameters (ADPs) and the lattice thermal conductivity of clathrate-like compounds. For many clathrate-like compounds, in which one of the atom types is weakly bound and "rattles" within its atomic cage, room temperature ADP information can be used to estimate the room temperature lattice thermal conductivity, the vibration frequency of the "rattler", and the temperature dependence of the heat capacity. Neutron data and X-ray crystallography data, reported in the literature, are used to apply this analysis to sevcral promising classes of thermoelectric materials.
\end{abstract}

\section{Introduction}

New bulk thermoelectric compounds are normally discovered with the aid of simple qualitative structure-property relationships. Most good thermoelectric compounds are narrow gap semiconductors composed of heavy elements with similar electronegativies. The crystal structures are usually of high symmetry (cubic, hexagonal, possibly tetragonal) and often contain a large number of atoms in the unit cell. Atomic displacement parameters (ADPs) measure the mean-square displacement amplitude of an atom about its equilibrium position in a crystal. In the description of a new crystalline compound, crystallographers normally tabulate the room temperature ADP values for each distinct atomic site in the structure ${ }^{1-4}$. The various ADP values thus comprise some of the first information that is known about a new crystalline compound. The value of the mean square atomic displacement can be due to the vibration of the atom or to static disorder. The effects that this parameter can have on various physical propertics, however, have not been widely recognized. In particular, ADPs are not normally used by solid state physicists or chemists as a guide in the search for new compounds with specific properties. ADPs are still regarded by many scientists as unreliable, since in many of the earliest structure determinations, ADPs often became repositories for much of the error in the structure refinement. In addition, crystallographers have not always reported ADP information using a consistent definition, ${ }^{1}$ adding further confusion as to the usefulness of ADPs. The purpose of this article is to illustrate that when properly determined, ADP information can be used as a guide in the search for crystalline materials with unusually low lattice thermal conductivities. These materials are of particular interest in the design of thermoelectric compounds with improved efficiencies. ${ }^{5.8}$ The analysis discussed below is restricted to clathrate-like compounds in which one of the atom types "rattles" about its equilibrium position in the crystal substantially more than the other atoms in the structure.
The compounds discussed here are the filled skutterudites $\left(\mathrm{R}_{\mathrm{x}} \mathrm{Co}_{4-\mathrm{y}} \mathrm{Fe}_{\mathrm{y}} \mathrm{Sb}_{12-\mathrm{z}} \mathrm{Sn}_{\mathrm{z}}\right.$ here $\mathrm{R}=\mathrm{La}, \mathrm{Ce}$, or $\left.\mathrm{Tl}, 0<(\mathrm{x}, \mathrm{y}, \mathrm{z})<1\right)$ and two new promising thermoelectric compounds $\mathrm{Tl}_{2} \mathrm{SnTe}_{5}$ and $\mathrm{Tl}_{2} \mathrm{GeTe}_{5} .{ }^{9}$ The filled skutterudites have been studied extensively for the past few years and exhibit excellent thermoelectric properties at elevated temperatures. ${ }^{10-16}$ In this structure, the "rattling" of the R atom in an oversized atomic cage is believed to be responsible for its low thermal conductivity. $\mathrm{Tl}_{2} \mathrm{SnTe}_{5}$ and $\mathrm{Tl}_{2} \mathrm{GeTe}_{5}$ exhibit more complicated tetragonal crystal structures, relative to the cubic filled skutterudites, but have the common features of weakly bound $\mathrm{Tl}$ atoms that reside at the centers of distorted, oversized cubic sites.

\section{Experimental}

Synthesis of the compounds is similar to that described in Ref. 14 , as are the details of the thermal conductivity measurements. Heat capacity data from $2-300 \mathrm{~K}$ were collected using a commercial system from Quantum Design. The ADP values were determined by powder neutron diffraction using ORNL's High Flux Isotope Reactor, and a neutron powder diffractometer equipped with a closed cycle helium refrigerator. For each sample the powder pattern was collected at several selected temperatures and refined using GSAS (General Structure Analysis System) software developed by Larson and von Dreele at the Los Alamos National Laboratory. As an additional check of the refinement methods, ADP values were also determined on single crystals of the filled skutterudites using a four-circle neutron diffractometer. Details of the refinement methods are described in Ref. 17. All of the ADP data have been converted to an isotropic, $U_{\text {iso }}$, value that has the dimensions of $\AA^{2}$. $U_{\text {iso }}$ measures the mean square displacement amplitude of the atom averaged over all directions. However, most ADP data reported in the literature are obtained using $x$-ray diffraction from small single crystals. Therefore, the examples of the structure property link between ADPs and lattice transport and dynamics in clathrate-like compounds shown in Table 1 and Fig.8, were calculated using reported $x$-ray ADP data. Obtaining quantitative ADP data using $x$-rays is in general more difficult than with neutrons. Absorption corrections are significant for $\mathrm{x}$-rays and must be made using either an empirical or analytical approach for the ADP values to be quantitative. As shown below, however, the Debye or Einstein temperatures depend on the square root of the ADP value, so that errors in an ADP value of $20-40 \%$ result in only a $9-18 \%$ percent variation in the Debye temperature. Both $x$-ray and neutron ADP data are available for the compounds $\mathrm{LaFe}_{4} \mathrm{Sb}_{12}$ and $\mathrm{Tl}_{2} \mathrm{SnTe}_{5}$, discussed below, and there is good agreement between the neutron and $\mathrm{x}$-ray ADP values. 


\section{Results and Discussion}

The simplest expression for the lattice thermal conductivity of a solid is given by an expression adapted from the kinetic theory of gases ${ }^{3}$ :

$$
\mathrm{K}_{\text {Lattice }}=1 / 3 \mathrm{C}_{\mathrm{V}} \mathrm{v}_{\mathrm{s}} \mathrm{d}
$$

where $C_{V}$ is the heat capacity per unit volume, $v_{S}$ is the velocity of sound and $d$ is the mean free path of the heat carrying phonons. In a more realistic treatment of lattice thermal conductivity, the mean free path (or relaxation time) and heat capacity depend on frequency, but for the present analysis $C_{v}$ depends only on temperature, while $v_{s}$ and $d$ are treated as scalars. It is well known to crystallographers that $\mathrm{ADP}$ data can be used to estimate the Debye temperature, $\Theta_{\mathrm{D}}$, $\mathrm{C}_{\mathrm{v}}$, and $\mathrm{v}_{\mathrm{S}}$ for any compound. ${ }^{4} \mathrm{U}_{\text {iso }}$ versus temperature can be calculated exactly for a cubic monatomic solid within the Debye model ${ }^{4}$. Fig. 1 illustrates how $\mathrm{U}_{\mathrm{iso}}$ depends on temperature for Debye temperatures of 100,200 , and $300 \mathrm{~K}$, and an atomic mass of $100 \mathrm{amu}$. The Debye temperature can be determined from the high temperature slope $\left(T>\Theta_{D}\right)$ of the ADP data, as indicated in the figure. The high temperature slope extrapolates to the origin (no zero-point energy offset). If just the room temperature value for $U_{\text {iso }}$ is used to extrapolate a slope to the origin, the error in the calculated Debye temperature is less than $10 \%$ if the actual Debye temperature is less than $600 \mathrm{~K}$. A natural extension of this

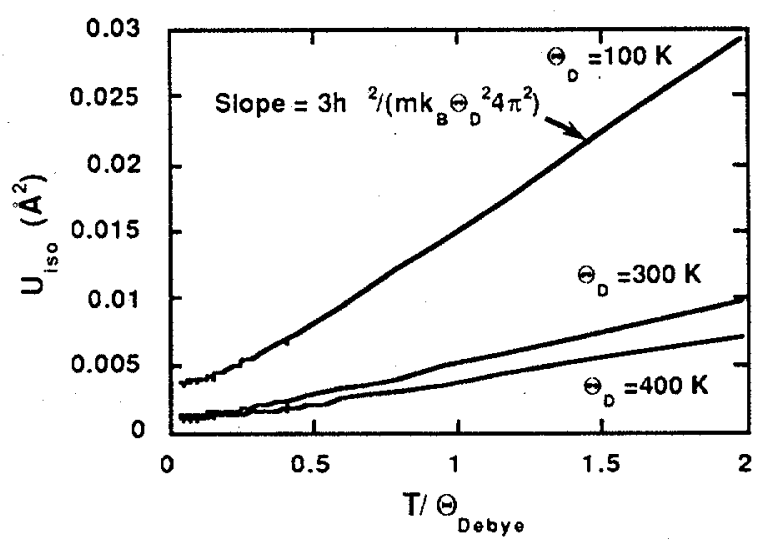

Fig. 1. Mean square displacement, $\mathrm{U}_{\mathrm{iso}}$, versus temperature scaled by the Debye temperature, $\Theta_{\mathrm{D}}$, for a monatomic cubic solid. $U_{\text {iso }}$ is shown for a solid composed of atoms with a mass of 100 amu for three different Debye temperatures. For temperatures greater than $\Theta_{D}, U_{i s o}$ is linear in $T$ with a slope as indicated.

analysis to a multi-element compound is accomplished by determining $U_{\text {iso }}$ for each element (or crystallographic site) and calculating the average $\mathrm{U}_{\text {iso }}$ and the average atomic mass. As an example, for the skutterudite $\mathrm{LaFc}_{4} \mathrm{Sb}_{12}$, the average room temperature $U_{i s}$ value is
$(0.014+0.0028 * 4+0.0049 * 12) / 17=0.0049 \AA^{2}$, and the average mass per atom is $107 \mathrm{amu}$. This results in a Debye temperature of $285 \mathrm{~K}$ ( $308 \mathrm{~K}$ measured $\left.{ }^{14}\right)$. From the Debye model the average velocity of sound is given by $v_{S}=\omega_{D} / K_{D}=$ $\left[\Theta_{D} \cdot k_{B} \cdot 2 \pi / h\right] /\left[6 \pi^{2} n\right]^{1 / 3}$, where $n$ is the number of atoms per unit volume. For $\mathrm{LaFe}_{4} \mathrm{Sb}_{12}$ this gives in $\mathrm{v}_{\mathrm{S}}=2750 \mathrm{~m} / \mathrm{s}(2923$ $\mathrm{m} / \mathrm{s}$ measured). ${ }^{14}$ The heat capacity is easily estimated once the Debye temperature is known and at room temperature is usually close to the classical Dulong and Petit value of 24.9 $\mathrm{J} / \mathrm{K}$-mole atoms.

The above analysis, using only the room temperature ADP values, can be used to estimate the Debye temperature, velocity of sound and heat capacity of any compound with a small amount of static disorder. Qualitatively, the effects of static disorder tend to displace the curves shown in Fig 1 upward by a constant amount. In all of the thermoelectric compounds that we have studied using neutron diffraction, static disorder is only a problem for alloys in which the crystallographic sites are only partially occupied. For the stochiometric compounds, $\mathrm{LaFe}_{4} \mathrm{Sb}_{12}, \mathrm{CeFe}_{4} \mathrm{Sb}_{12}, \mathrm{Tl}_{2} \mathrm{SnTe}_{5}$, and $\mathrm{Tl}_{2} \mathrm{GeTe}_{5}$, the temperature dependent $\mathrm{ADP}$ values all extrapolate to values close to the origin. This is illustrated in Fig. 2 for $\mathrm{Tl}_{2} \mathrm{SnTe}_{5}$. The $\mathrm{Tl}$ at site (1) in the $\mathrm{Tl}_{2} \mathrm{SnTe}_{5}$ crystal structure is weakly bound at the center of a distorted cube, and clearly "rattles" more about its equilibrium position than the other atoms in the structure.

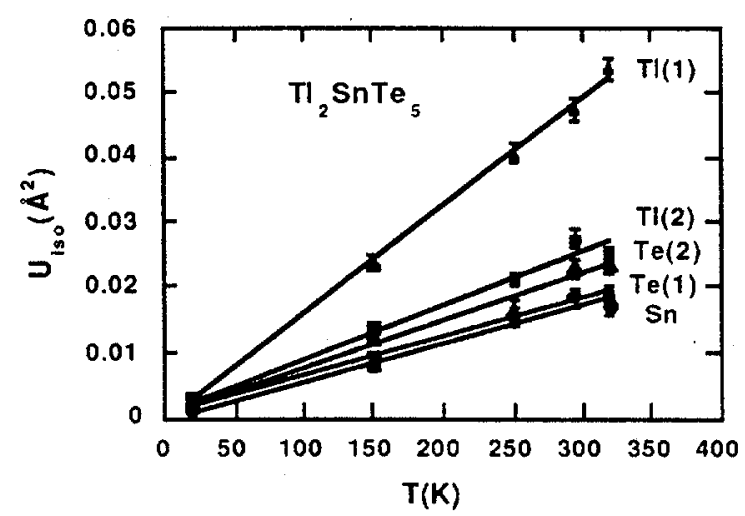

Fig. 2. ADP versus temperature for $\mathrm{Tl}_{2} \mathrm{SnTe}_{5}$ determined from powder neutron diffraction. Note the larger ADP values for $\mathrm{Tl}$ at site 1 in the crystal structure. These $\mathrm{Tl}$ "rattle" in oversized atomic cages.

To estimate the lattice thermal conductivity using Eq. [1], however, requires a value for $\mathrm{d}$. The phonon mean free path (or scattering rate) in the clathrate-like compounds is determined by the various scattering mechanisms in the crystal, such as acoustic phonons, grain boundaries, electronphonon interactions, static defects, voids and "rattlers". Phonon scattering in these materials appears to be dominated by the "rattlers" ${ }^{18}$. This is illustrated in Figure 3, which shows the large reduction in thermal conductivity that results as $\mathrm{Tl}$ is added to $\mathrm{CO}_{4} \mathrm{Sb}_{12}$. In the compound shown, $\mathrm{Tl}_{0.22} \mathrm{Co}_{4} \mathrm{Sb}_{12}, \mathrm{Tl}$ fills $22 \%$ of the available cages in the structure. $\mathrm{Tl}_{2} \mathrm{SnTe}_{5}$ and 
$\mathrm{Tl}_{2} \mathrm{GeTe}_{5}$ exhibit a more complicated crystal structure, relative to the cubic filled skutterudites, but have the common features of weakly bound $\mathrm{Tl}$ atoms that reside at the center of a distorted, oversized cubic site. As can be seen from Figure 4, this results in a remarkably low thermal conductivity, much lower at room temperature than that of vitreous silica.

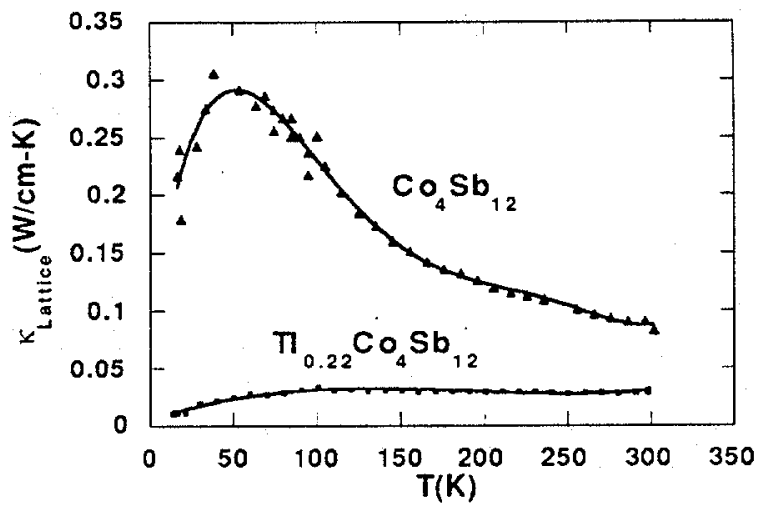

Fig. 3 Lattice thermal conductivity versus temperature for hot-pressed ceramics of $\mathrm{Co}_{4} \mathrm{Sb}_{12}$ and $\mathrm{Tl}_{0.22} \mathrm{Co}_{4} \mathrm{Sb}_{12}$. The Wiedemann-Franz law has been used to estimate and subtract the electronic portion of the thermal conductivity. The lines through the data are guides to the eye.

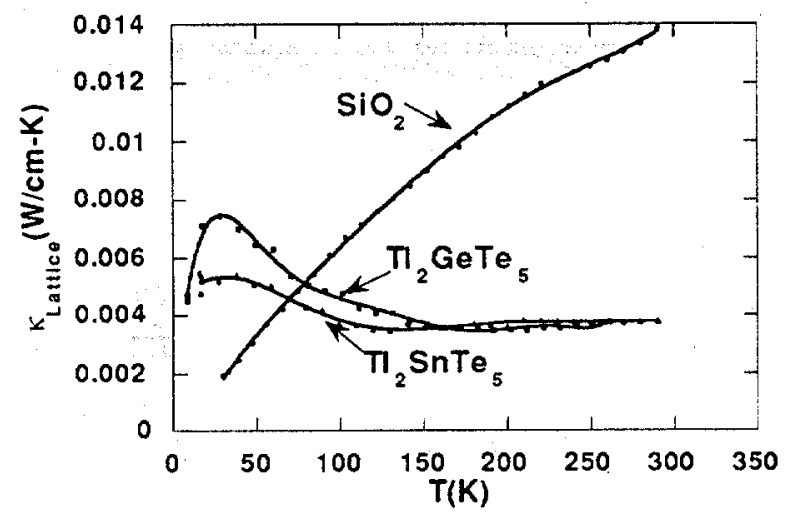

Fig. 4 Lattice thermal conductivity versus temperature for vitreous silica, $\mathrm{Tl}_{2} \mathrm{SnTe}_{5}$ and $\mathrm{Tl}_{2} \mathrm{GeTe}_{5}$. The WiedemannFranz law has been used to estimate and subtract the electronic portion of the thermal conductivity. The lines through the data are guides to the eye.

If the "rattlers" are truly localized vibrations (or Einstein oscillators), as suggested by Keppens et al. ${ }^{19}$, then the heat carrying phonon mean free path should be a function of the distance between the "rattlers" in the crystal. The simplest estimate of the phonon mean free path is therefore the distance between the "rattlers". Physically this implies that the resonant scattering of the acoustic phonons by the "rattlers" is so strong that $\mathrm{d}$ attains a minimum value given by the average distance between "rattlers". The scattering of acoustic phonons by the "rattlers" will clearly be a maximum when the acoustic phonon and "rattling" frequency are equal. ${ }^{20}$ However, even on resonance, it seems physically unlikely that d could be less than the "rattler" separation distance. At room temperature, this argument works well for the filled skutterudites such as $\mathrm{LaFe}_{4} \mathrm{Sb}_{12 .}{ }^{14}$ Using the measured thermal conductivity, heat capacity and an average value for the velocity of sound ${ }^{14}$ yields a mean free path of $d=9 \AA$. The nearest neighbor distance of the $\mathrm{La}$ atoms in $\mathrm{LaFe}_{4} \mathrm{Sb}_{12}$ is 7.9 $\AA$. If the role of other scattering mechanisms can be minimized, this simple model would suggest that the thermal resistivity should vary as $x^{1 / 3}$, where $x$ is the concentration of rattlers (the average spacing between "rattlers" varies as $\left.\mathrm{x}^{-1 / 3}\right)$. This behavior was carefully examined in two families of filled skutterudites: $\mathrm{Tl}_{x} \mathrm{Co}_{4} \mathrm{Sb}_{12 . y} \mathrm{Sn}_{y}$ and $\mathrm{Tl}_{x} \mathrm{Co}_{4 . y} \mathrm{Fe}_{y} \mathrm{Sb}_{12}(0<$ $(x, y)<1)$. Within the uncertainty of the measured thermal resistance data, an $x^{1 / 3}$ law provides a surprisingly good description of the data as the concentration of the $\mathrm{Tl}$ "rattlers" is varied between 0 and 1 (Fig. 5). For values of $x>0.5$, we estimate that at least $70 \%$ of the room temperature thermal resistance is due to scattering by the Tl "rattlers". We caution, however, that at low $\mathrm{Tl}$ concentrations we would expect, on general theoretical grounds, the increase in the thermal resistivity to be linear in the $T 1$ concentration, $x$. How this behavior saturates at higher concentrations is not understood. The data shown in Fig. 5 simply suggest that replacing $d$ in Eq. [1] by the average "rattler" separation is plausible. The real test of this hypothesis is whether this analysis results in good estimates of the room temperature lattice thermal conductivity for a variety of clathrate-like compounds.

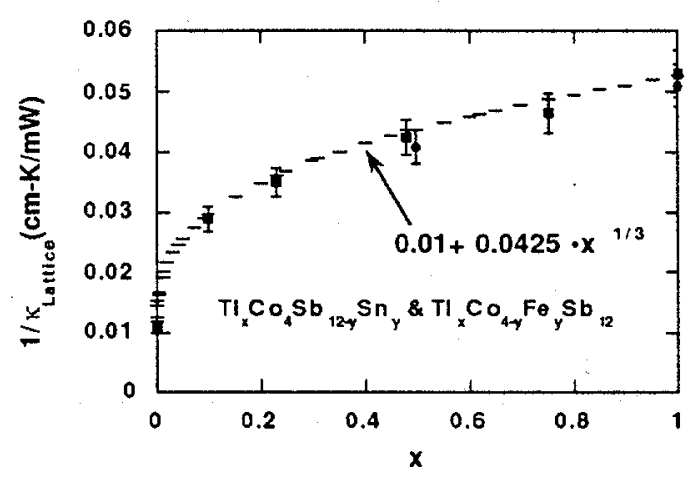

Fig. 5. Variation of the room temperature thermal resistivity vs. the fraction of cages filled with the "rattler" Tl. In the filled skutterudite structure, the average separation distance between $\mathrm{Tl}$ "rattlers" varies as $\mathrm{x}^{-1 / 3}$. For all but the two lowest concentrations of $\mathrm{Tl}, \mathrm{x} \approx \mathrm{y}$. The square (circle) symbols refer to charge compensation with $\mathrm{Sn}(\mathrm{Fe})$. The electronic contribution to the thermal conductivity has been subtracted using the Wiedemann-Franz law.

Estimates of the room temperature lattice conductivity, determined using published $\mathrm{x}$-ray data, are compared in Table 1 with the measured values for three classes of promising thermoelectric materials. The estimates are surprisingly close to the measured values. 
Data similar to that shown in Fig. 5 have been previously reported for $\mathrm{La}$ and $\mathrm{Ce}$ rattlers in the filled skutterudite structure. The present data agree reasonably well with the previous La data reported by Nolas et al. ${ }^{18}$, particularly at the low La concentrations. The peak in the thermal resistivity for Ce filling fractions near $x=0.6-0.7$ reported by Meisner et. al.

${ }^{21}$ was not observed in the present work.

If the "rattler" represents a localized vibrational mode that resonantly scatters acoustic phonons, the dynamics of the rattler are more appropriately described by a quantized harmonic oscillator (Einstein oscillator) rather than the Debye model. This implies that all of the "rattlers" vibrate at about the same frequency and are poorly coupled to the lattice dynamics of the rest of the solid. Of course there has to be some coupling, otherwise the "rattlers" couldn't scatter acoustic phonons! This suggests that a better starting point for understanding the low energy lattice dynamics of these types of materials is to treat the "rattlers" as Einstein oscillators and the other atoms as part of a Debye solid. The decomposition of these solids into an Einstein part and a Debye part does not significantly affect the estimate of the room temperature lattice thermal conductivity, discussed above, since at high temperatures the Debye and Einstein models both tend toward the classical value for the heat capacity. The Einstein mode, however, will significantly affect the temperature dependence of the heat capacity at low temperatures, and will result in a peak in the phonon density of states. This mode should also be visible in a variety of spectroscopic measurements.

The room temperature ADP values for the "rattler" can also be used to estimate the Einstein frequency of the "rattler". The mean square displacement amplitude, $\left\langle u^{2}\right\rangle$ of a quantized harmonic oscillator ${ }^{2}$ is given by:

$$
\mathrm{U}_{\text {iso }}=\left\langle\mathrm{u}^{2}\right\rangle=h /\left(8 \mathrm{v}^{2} \mathrm{~m}\right) \cdot \operatorname{coth}\left(h v / 2 \kappa_{\mathrm{B}} \mathrm{T}\right)
$$

where $m$ is the mass of the rattler and $v$ is the frequency of vibration. The temperature dependence of Eq. [2] is qualitatively similar to that shown in Fig. 1 with $\Theta_{R}=h v / k_{B}$ replacing $\Theta_{D}$. At high temperatures, where hv $<2 \mathrm{k}_{\mathrm{B}} \mathrm{T}$, Eq. [2] reduces to the classical expression $U_{i s o}=k_{B} T / K$ where $K$ is the force constant of the oscillator; $K=m(2 \pi v)^{2}$. As was the case for the Debye model, the room temperature ADP data can be used to estimate the Einstein temperature of the "rattler" only if the static disorder is small and the Einstein temperature is less than about $300 \mathrm{~K}$.

The temperature dependence of the ADP's are shown in Fig. 6 for the skutterudite alloy $\mathrm{Tl}_{0.22} \mathrm{Co}_{4} \mathrm{Sb}_{12}$ in which $22 \%$ of the available voids in $\mathrm{CoSb}_{3}$ are filled with $\mathrm{Tl}$. The slope of the $\mathrm{Tl}$ ADP data implies an Einstein temperature for the $\mathrm{Tl}$ atoms of $53 \mathrm{~K}$.
Heat capacity measurements were made on $\mathrm{Tl}_{0.22} \mathrm{CO}_{4} \mathrm{Sb}_{12}$ and $\mathrm{Co}_{4} \mathrm{Sb}_{12}$. The heat capacity difference between these two compounds gives the contribution due to the $\mathrm{Tl}$ atoms. The $\mathrm{Tl}$ contribution is accurately described by a simple quantized harmonic oscillator (Einstein model) with a characteristic temperature of $55 \pm 2 \mathrm{~K}$ (Fig. 7), in excellent agreement with the value of $53 \mathrm{~K}$ predicted by an analysis of the ADP data in Fig 6 .

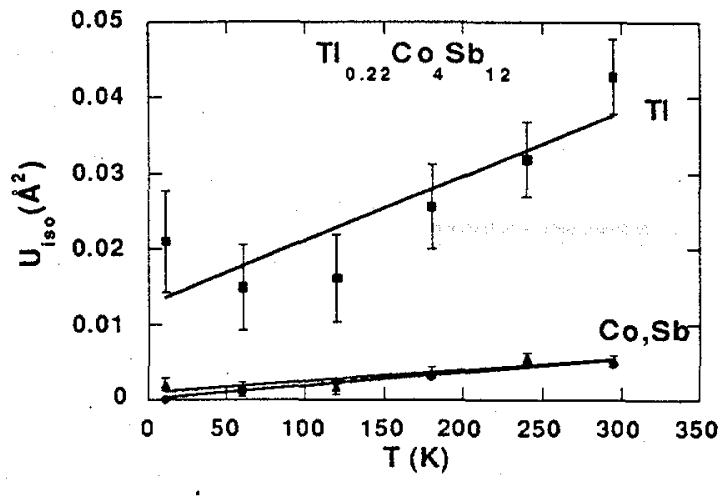

Fig. 6 Atomic displacement parameters versus temperature for $\mathrm{Tl}_{0.22} \mathrm{Co}_{4} \mathrm{Sb}_{12}$. The lines shown are a least squares fit to the data.

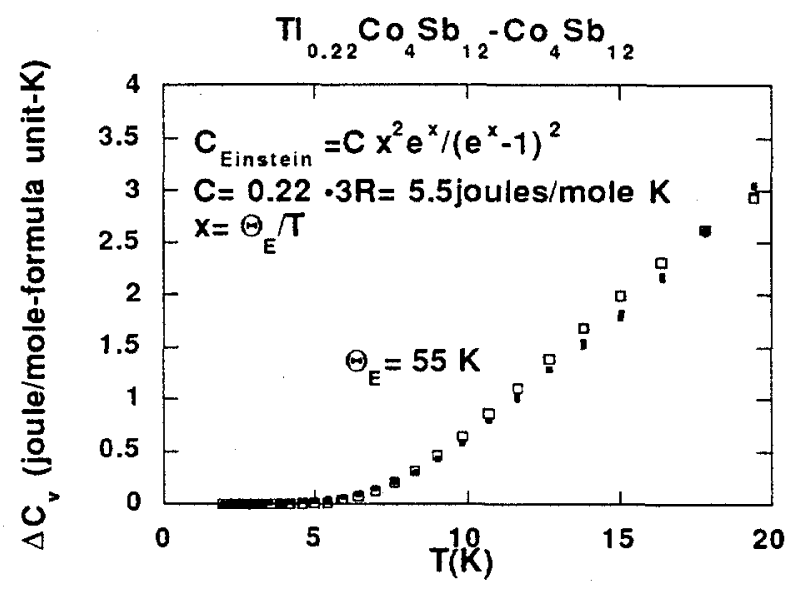

Fig. 7 Difference in heat capacity between on $\mathrm{Tl} \quad{ }_{0.22} \mathrm{Co}_{4} \mathrm{Sb}_{12}$ and $\mathrm{Co}_{4} \mathrm{Sb}_{12}$. The data is accurately described by quantized harmonic oscillator (Einstein model) with an Einstein temperature of $55 \mathrm{~K}$. A small electronic heat capacity contribution was subtracted from the $\mathrm{Tl}_{0.22} \mathrm{Co}_{4} \mathrm{Sb}_{12}$ data.

Comparisons between the Einstein temperatures determined using the room temperature ADP data, with the Einstein temperatures determined using other techniques (inelastic neutron scattering, heat capacity and elastic constant data) are shown in Table 1. 
Table 1. Comparison between thermodynamic quantities estimated using room temperature x-ray crystallography data (ADP columns) and the same quantities measured either directly or inferred from another technique.

\begin{tabular}{|c|c|c|c|c|c|c|}
\hline & \multicolumn{2}{|c|}{$\mathrm{LaFe}_{4} \mathrm{Sb}_{12}$} & \multicolumn{2}{|c|}{$\mathrm{Tl}_{2} \mathrm{SnTe}_{5}$} & \multicolumn{2}{|c|}{$\mathrm{Ba}_{8} \mathrm{Ga}_{16} \mathrm{Ge}_{30}$} \\
\hline & $\mathrm{ADP}^{22}$ & Other ${ }^{14}$ & $\mathrm{ADP}^{23}$ & Other & $\mathrm{ADP}^{24}$ & Other ${ }^{26}$ \\
\hline$\Theta_{\mathrm{D}}(\mathrm{K})$ & 299 & 305 & 159 & 160 & 275 & 300 \\
\hline$\Theta_{\mathrm{E}}(\mathrm{K})$ & 79 & 80 & 38 & 30 & 51 & 50 \\
\hline $\mathrm{v}_{\mathrm{s}}(\mathrm{m} / \mathrm{s})$ & 2886 & 3007 & 1488 & -- & 2123 & $\therefore$ \\
\hline$d(\AA)$ & 7.9 & 9 & 6.45 & -- & 6.3 & $\cdots$ \\
\hline $\mathrm{K}(\mathrm{W} / \mathrm{cm}-\mathrm{K})$ & 0.014 & 0.017 & 0.004 & 0.004 & 0.008 & 0.020 \\
\hline
\end{tabular}

As an example of the surprisingly good results that can be obtained using room temperature $x$-ray ADP values, the heat capacity of $\mathrm{Tl}_{2} \mathrm{SnTe}_{5}$ was predicted, with no adjustable parameters, and then measured. An Einstein contribution was used to describe the $\mathrm{Tl}$ "rattler" (the $\mathrm{Tl}$ at site 1 in the crystal structure, see Fig. 2) with an Einstein temperature of $38 \mathrm{~K}$ and an amplitude of $1 / 8 \cdot 3 \mathrm{R}$, where $\mathrm{R}$ is the gas constant. The $1 / 8$ is because only 1 out of the 8 atoms per formula units is a "rattler". For the rest of the atoms a Debye heat capacity was used with a Debye temperature of $159 \mathrm{~K}$ and an amplitude of $7 / 8 \cdot 3 R$. A comparison between the measured and predicted heat capacity is shown in Fig. 8. The agreement is remarkable. At temperatures below $15 \mathrm{~K}$ (Fig. 8 inset), however, the data indicates that an Einstein temperature of about $30 \mathrm{~K}$ provides a better description of the heat capacity data than does the value of $38 \mathrm{~K}$ determined from the room temperature ADP data.

\section{Summary}

In summary, we have shown that room temperature ADP data can be used to identify new compounds with unusually low lattice thermal conductivities. For large classes of clathratelike compounds, the room temperature ADP data reported as part of crystal structure determinations can be used to estimate the Debye temperature, velocity of sound, mean free path of phonons, lattice thermal conductivity, heat capacity, as well as the Einstein frequency of the "rattler". The Si and Ge clathrate compounds, such as recently reported by Nolas et al. ${ }^{25,27}$, are excellent candidate materials to further test some of these simple ideas.

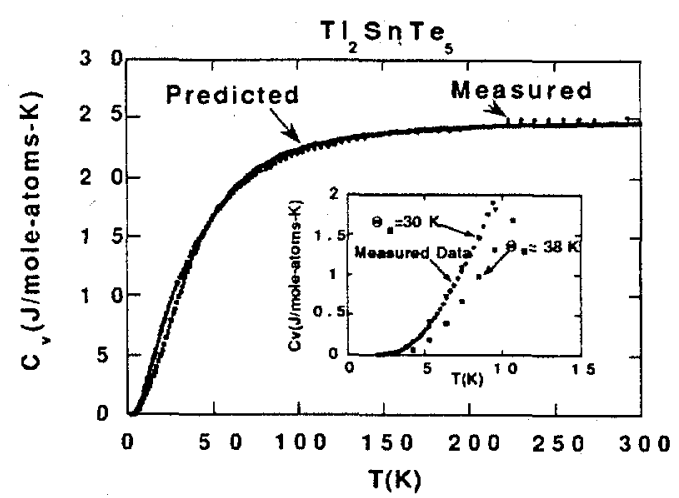

Fig. 8 Predicted and measured heat capacity for $\mathrm{Tl}_{2} \mathrm{SnTe}_{5}$. The predicted heat capacity was determined using the published room temperature x-ray crystallography ADP data of Ref. 24. There were no adjustable parameters. Inset shows the predicted and measured heat capacity. At low temperatures an Einstein temperature of $30 \mathrm{~K}$ provides a better description of the data than the predicted value of $38 \mathrm{~K}$.

\section{Acknowledgments}

It is a pleasure to acknowledge useful discussions with Glen Slack, George Nolas, Gerald Mahan, and Mercouri Kanatzidis. Research was sponsored in part by a Cooperative Research and Development Agreement with Marlow Industries and in part by the Division of Materials Sciences, U.S. Department of Energy Contract No. De-ACO5960R22464. Oak Ridge National Laboratory is managed by the Lockheed Martin Energy Research Corporation for the U.S. Department of Energy.

\section{References}

1. W. F. Kuhs, Acta Cryst. A48,80 (1992).

2. J.D. Dunitz, V. Schomaker, and K. N. Trueblood, J. Phys. Chem. 92, 856 (1988).

3. C. Kittel, Introduction to Solid State Physics, Third Edition (John Wiley and Sons, New York 1968), pp. 69 70, p. 186.

4. B. T. M. Willis and A. W. Pryor, Thermal Vibrations in Crystallography (Cambridge University Press, London 1975).

5. G. D. Mahan, B. C. Sales, and J. W. Sharp, Physics Today, 50, No. 3, 42 (1997).

6. G. A. Slack in CRC Handbook of Thermoelectrics, edited by D. M. Rowe (Chemical Rubber, Boca Raton, FL, 1997) pp. 407-440.

7. H. J. Goldsmid, Electronic Refrigeration (Pion Limited, London 1986).

8. B. C. Sales, MRS Bulletin 23, 15 (1998).

9. J. W. Sharp, B. C. Sales, B. C. Chakoumakos and D. Mandrus, Appl. Phys. Lett., 74, 3794 (1999).

10. D. T. Morelli and G. P. Meisner, J. Appl. Phys. 77, 3777 (1995).

11. B. C. Sales, D. Mandrus, and R. K. Williams, Science 272, 1325 (1996) 
12. J. -P. Fleurial, A. Borshchevsky, T. Caillat, D. T. Morelli, and G. P. Meisner, in Proceedings of the Fifteenth Conference on Thermoelectrics, Pasadena CA (IEEE Piscataway, NJ, 1996) p. 91.

13. G. S. Nolas, G. A. Slack, D. T. Morelli, T. M. Tritt, and A. C. Ehrlich, J. Appl. Phys. 79, 4002 (1996).

14. B. C. Sales, D. Mandrus, B. C. Chakoumakos, V. Keppens, and J. R. Thompson, Phys. Rev. B. 56, 15081 (1997).

15. B. Chen, J. X. Xu, C. Uher, D. T. Morelli, G. P. Meisner, J. P. Fleurial, T. Caillat, and A. Borshchevshy, Phys. Rev. B 55, 1476 (1997).

16. N. R. Dilley, E. J. Freeman, E. D. Bauer, and M. B. Maple, Phys. Rev. B. 58, 6287 (1998).

17. B. C. Chakoumakos, B. C. Sales, D. Mandrus, and V. Keppens, Acta. Cryst. B 55, 341 (1999).

18. G. S. Nolas, J. L. Cohn, and G. A. Slack, Phys. Rev. B. 58, 164 (1998).

19. V. Keppens, D. Mandrus, B. C. Sales, B. C. Chakoumakos, P. Dai, R. Coldea, M. B. Maple, D. A. Gajewski, E. J. Freeman, and S. Bennington, Nature 395, 876 (1998).

20. R. O. Pohl Phys. Rev. Lett. 8, 12 (1962)

21. G. P. Meisner, D. T. Morelli, S. Hu, J. Jong, and C. Uher, Phys. Rev. Lett. 80, 3551 (1998).

22. D. J. Braun and W. Jeitschko, J. Less Common Metals 72, 147 (1988).

23. V. Agafonov, B. Legendre, N. Rodier, J. M. Cense, E. Dichi, and G. Kra, Acta. Cryst. C47. 850 (1991).

24. B. Eisenmann, H. Schafer, and R. Zagler, $J$. Less. Common Metals 118, 43 (1986).

25. G. S. Nolas, J. L. Cohn, G. A. Slack, and S. B. Schujman, Appl. Phys. Lett. 73, 178 (1998).

26. B. C. Sales, unpublished data.

27. J. L. Cohn, G. S. Nolas, V. Fessatidis, T. H. Metcalf, G. A. Slack, Phys. Rev. Lett., 82, 779 (1999). 